\title{
Dementia as a core clinical feature of a patient with aceruloplasminemia
}

\author{
Farzad Ashrafi ${ }^{1}$, Mehri Salari ${ }^{2}$, Fatemeh Nouri $^{2}$, and fatemeh shiravi ${ }^{2}$ \\ ${ }^{1}$ Shohada-e Tajrish Hospital \\ ${ }^{2}$ Shahid Beheshti University of Medical Sciences
}

November 10, 2021

\begin{abstract}
Aceruloplasminemia is an autosomal recessive disease, caused by systemic iron accumulation due to mutations in the Ceruloplasmin gene. We report two Iranian siblings who have diagnosed with aceruloplasminemia. Based on our researches, dementia hasn't been published as the first neurological feature while one of them was presented with pure dementia
\end{abstract}

\section{Introduction}

Aceruloplasminemia was firstly defined in 1987 as a rare adult-onset, an autosomal recessive disorder which is caused by mutations in the ceruloplasmin gene. [1-3]

As a consequence of this mutation iron accumulates all over the body, mostly in brain, retina, pancreas, and liver, which causes neurologic disturbance $(68 \%)$, retinal degeneration $(76 \%)$, and diabetes $(70 \%)$, as main features. $[4,5]$

The iron deposition involves the dentate nuclei of the cerebellum, striatum, and thalamus, and is demonstrated in magnetic resonance imaging (MRI).[6]

The disease can start at different ages and by various features.[4] The patients may experience diabetes in the third to the fifth decade, the retinal disorder in the second decade, and neurological problems in the forth to the sixth decade of life. Neurological manifestation can be ataxia (71\%), Parkinsonism (20\%) cognitive dysfunction (60\%) and involuntary movements (64\%) like tremors, chorea and blepharospasm. The retinal disorder is early beginning macular degeneration rather than diabetic retinopathy. [5]

Aceruloplasminemia is detected with specific MRI findings, quite one amongst the above clinical manifestations and typical results on laboratory tests, such as microcytic anemia, lack of serum ceruloplasmin, low serum copper and transferrin saturation, and increased ferritin. $[4,7,8]$

In this paper, we describe a family with two affected siblings with aceruloplasminemia, which dementia was the sole clinical manifestation in one of them.

\section{Case report}

Case 1:

A 64-year-old woman was referred to our movement disorder clinic with four years history of bradykinesia and rigidity. She was born as a first child of consanguineous parents and has history of diabetes mellitus, myelodysplastic syndrome, and gout for 15 years. 
On examination, she had generalized bradykinesia and rigidity without tremor, slow saccadic eye movements, limbs dysmetria and ataxic gait. Other neurological examination including, cognitive, sensory, and motor function were unremarkable.

On follow-up, after 2 years, she became wheelchair-bound due to severe gait freezing, cognitively declined (MOCA: 19), and tremor was added to her clinical manifestations. In addition, at that time she had severe aggression, agitation, and visual hallucination.

The evaluation showed microcytic anemia, low serum ceruloplasmin, copper and transferrin saturation, and increased ferritin. (Table 1) Brain MRI detected abnormal signal intensity of the cerebellum, basal ganglia, and midbrain. (Figure A, B) According to imaging findings and other laboratory assessments, the diagnosis of aceruloplasminemia was suspected. Genetic analysis was requested. The direct DNA Sequencing revealed homozygote pathogenic variant defined as $(\mathrm{c} .2425+1 \mathrm{G}>\mathrm{C})$ in splice region of exon 13 of $\mathrm{CP}$ gene. DNA was extracted from the patient sample using salting-out technique. PCR was used to intensify the indicated exons plus extra flanking intronic or other non-coding sequences. Subsequently cleaning of the PCR supplies, cycle sequencing was carried out using the ABI Big Dye Terminator v.3.0 kit. Products were resolved by electrophoresis on an ABI 3130 capillary sequencer. Sequencing was accomplished separately in both the forward and reverse directions.

\section{Case 2:}

The 56-year-old sibling had had cognitive decline for 8 years, and Alzheimer's disease had been diagnosed. . He has a history of diabetes mellitus (DM) since the age of 28. On examination, he had mostly attention, concentration, memory, executive dysfunction, and his MOCA was 5. Other examinations including, cerebellar, pyramidal, extrapyramidal, eye movements, and sensory function were unremarkable.

According to his positive family history, more workup showed microcytic anemia, elevated ferritin, and low serum ceruloplasmin. (Table 1)

The brain MRI revealed low signal intensity on the T2/fluid attenuated inversion recovery weighted image. (Figure C, D)

After careful history taking, it was showed that the apart that case 1, other siblings have diabetes, plus the younger brother has a high ferritin level and mild cognitive dysfunction without other neurological findings.

The family history, laboratory and imaging results were leading to the diagnosis of aceruloplasminemia, therefore, the genetic study was asked and showed homozygote pathogenic variant defined as (c.2425+1G>C) in splice region of exon 13 of $\mathrm{CP}$ gene.

\section{Discussion}

Ceruloplasmin which is mostly synthetized in liver cells has a crucial role within the iron metabolism by oxidation of $\mathrm{Fe} 2+$ to $\mathrm{Fe} 3+$. [8] This protein's gene is positioned in chromosome 3q25, and according to human gene mutation databank more than $40 \mathrm{CP}$ gene variant has been described; previous studies failed to show any correlations between the genotypes and, phenotypes. $[9,10]$ In our patients by direct DNA Sequencing we revealed a unique variant which is $(c .2425+1 G>C)$ in splice region of exon 13 of CP gene.

CP mutation causes iron to accumulate all over the body that provides free radicals and lipoperoxidation supplies that cause oxidative stress and eventually organ damage. [9]

According to prior researches, the initial neurological presentation varies in different races as an example in Japanese patients, the primary neurological manifestations mostly are movement disorders, and cognitive dysfunction will be added in several years. Although, in other races such as Caucasians around $50 \%$ of patients at first presented with cognitive disorders or psychiatric complaints such as depression, anxiety and apathy or a mixture of non-motor and motor manifestations.[11]. Based on these researches, dementia has not been published as the sole neurological feature while we reported a case which was presented with pure dementia without any other neurological findings which hitherto no other neurological symptoms have 
been added. Forasmuch as there are the first Iranian cases of Aceruloplasminemia no previous research has been done.

\section{Acknowledgment:}

This research did not receive any specific grant from funding agencies in the public, commercial, or not-forprofit sectors.

\section{Authors list:}

Farzad Ashrafi, MDFunctional Neurosurgery Research Center, Shohada-e-Tajrish Neurosurgical Center of Excellence, Shahid Beheshti University of Medical Sciences, Tehran, Iran. Email: farzad.ashrafi@gmail.com

Mehri Salari, MD

Functional Neurosurgery Research Center, Shohada Tajrish Neurosurgical Center of Excellence, Shahid Beheshti University of Medical Sciences, Tehran, Iran. Email: mehri.salari@gmail.com

Fatemeh Nouri *Student Research Committee, School of Medicine, Shahid Beheshti university of Medical Science, Tehran, Iran. Email: mel29.nor@gmail.com

Fatemeh Shiravi*Student Research Committee, School of Medicine, Shahid Beheshti university of Medical Science, Tehran, Iran. Email: fatemeh.sh1996@gmail.com

*These authors contributed equally to the study and share corresponding authorship.

\section{Corresponding author:}

Fatemeh Nouri * Student Research Committee, School of Medicine, Shahid Beheshti university of Medical Science, Tehran, Iran. E-Mail: mel29.nor@gmail.com

Tel: $+98-21-22724214$

ORCID: 0000-0001-6671-3845

Fatemeh shiravi* Student Research Committee, School of Medicine, Shahid Beheshti university of Medical Science, Tehran, Iran.

E-Mail: fatemeh.sh1996@gmail.com Tel: +98-21-22724214 ORCID: 0000-0003-0751-7478

\section{Author Contributions:}

All authors have made substantial contributions to the conception and design and acquisition of data and interpretation of data, and all of them have been involved in drafting the manuscript or revising it critically for important intellectual content. All the listed authors have given final approval of the version to be published. They all agreed to be accountable for all aspects of the work in ensuring that questions related to the accuracy or integrity of any part of the work are appropriately investigated and resolved.

Farzad Ashrafi, MD: Conception, Organization

Mehri Salari, MD: Conception, Organization, Execution, Review and Critique.

Fatemeh Nouri *: Writing of the first draft, Corresponding author

Fatemeh Shiravi*: Writing of the first draft, Corresponding author

*These authors contributed equally to the study and share corresponding authorship.

\section{References}

1. Piperno A, Alessio M. 2018. Aceruloplasminemia: Waiting for an Efficient Therapy. Front Neurosci.12:18. 
2. Marchi G, Busti F, Zidanes AL, et al . 2019. Aceruloplasminemia: A severe neurodegenerative disorder deserving an early diagnosis. Front. Neurosci..13:1-8.

3. Doyle A, Rusli F, Bhathal P . 2015. Aceruloplasminaemia:A rare but important cause of iron overload. BMJ Case Rep:1-4.

4. Yamamura A, Kikukawa Y, Tokunaga K, et al . 1905-1910. Pancytopenia and myelodysplastic changes in aceruloplasminemia: A case with a novel pathogenic variant in the ceruloplasmin gene. Intern Med 2018. $57:$.

5. Miyajima H, Hosoi Y . Aceruloplasminemia Summary Genetic counseling. University of Washington, Seattle.2019.

6. Hayashida M, Hashioka S, Miki H, et al . 2016. Aceruloplasminemia with psychomotor excitement and neurological sign was improved by minocycline (Case Report). Med (United States). 95:1-7.

7. Miyajima H . 2015. Aceruloplasminemia. Neuropathology. 35:83-90.

8. Roberti M do RF, Filho HMB, Gonçalves CH, Lima FL . 2011. Aceruloplasminemia: A rare disease diagnosis and treatment of two cases. Rev Bras Hematol Hemoter. 33:389-392.

9. Watanabe M, Ohyama K, Suzuki M, et al . 2018. Aceruloplasminemia with abnormal compound heterozygous mutations developed neurological dysfunction during phlebotomy therapy. Intern Med. 57:2713-2718.

10. Tai M, Matsuhashi N, Ichii O, et al . 2014. Case of presymptomatic aceruloplasminemia treated with deferasirox. Hepatol Res. 44:1253-1258.

11. Vroegindeweij LHP, Langendonk JG, Langeveld M, et al . 2017. New insights in the neurological phenotype of aceruloplasminemia in Caucasian patients. Park Relat Disord. 36:33-40.

\begin{tabular}{llll}
\hline & Case 1 & Case 2 & Normal Range \\
\hline white blood cell $(/ \mathrm{uL})$ & 5900 & 7900 & $4000-10800$ \\
red blood cell $(\times 106 / \mathrm{uL})$ & 4.64 & 5.11 & $3.8-5.2$ \\
Hemoglobin $(\mathrm{g} / \mathrm{dL})$ & 10.6 & 11.90 & $11.5-16$ \\
Hematocrit $(\%)$ & 34.5 & 38.80 & $32-46$ \\
mean corpuscular volume (fL) & 74.4 & 75.90 & $80-96$ \\
mean corpuscular hemoglobin (pg) & 22.8 & 23.30 & $24-35$ \\
Mean corpuscular hemoglobin concentration $(\%)$ & 30.7 & 30.70 & $28-38$ \\
Red blood cell distribution width $(\%)$ & 19 & 17.50 & $<15$ \\
erythrocyte sedimentation rate $(\mathrm{mm} / \mathrm{hr})$ & 10 & 10 & $0-30$ \\
Fasting Blood Sugar $(\mathrm{mg} / \mathrm{dL})$ & 90 & 307 & $70-115$ \\
Urea $(\mathrm{mg} / \mathrm{dL})$ & 21 & 29 & $15-45$ \\
blood urea nitrogen $(\mathrm{mg} / \mathrm{dL})$ & 9.8 & 9.9 & $4-23$ \\
Creatinine $(\mathrm{mg} / \mathrm{dL})$ & 0.8 & 0.8 & $0.5-1.3$ \\
Serum copper $(\mu \mathrm{d} / \mathrm{dL})$ & 9 & 25 & $80-155$ \\
Ceruloplasmin $(\mathrm{mg} / \mathrm{dL})$ & 6.4 & 6 & $15-60$ \\
Ferritin $(\mu \mathrm{g} / \mathrm{L})$ & 1008.8 & 1460 & $5-73.3$ \\
\hline
\end{tabular}

Table:

Laboratory Examinations

Figure: 

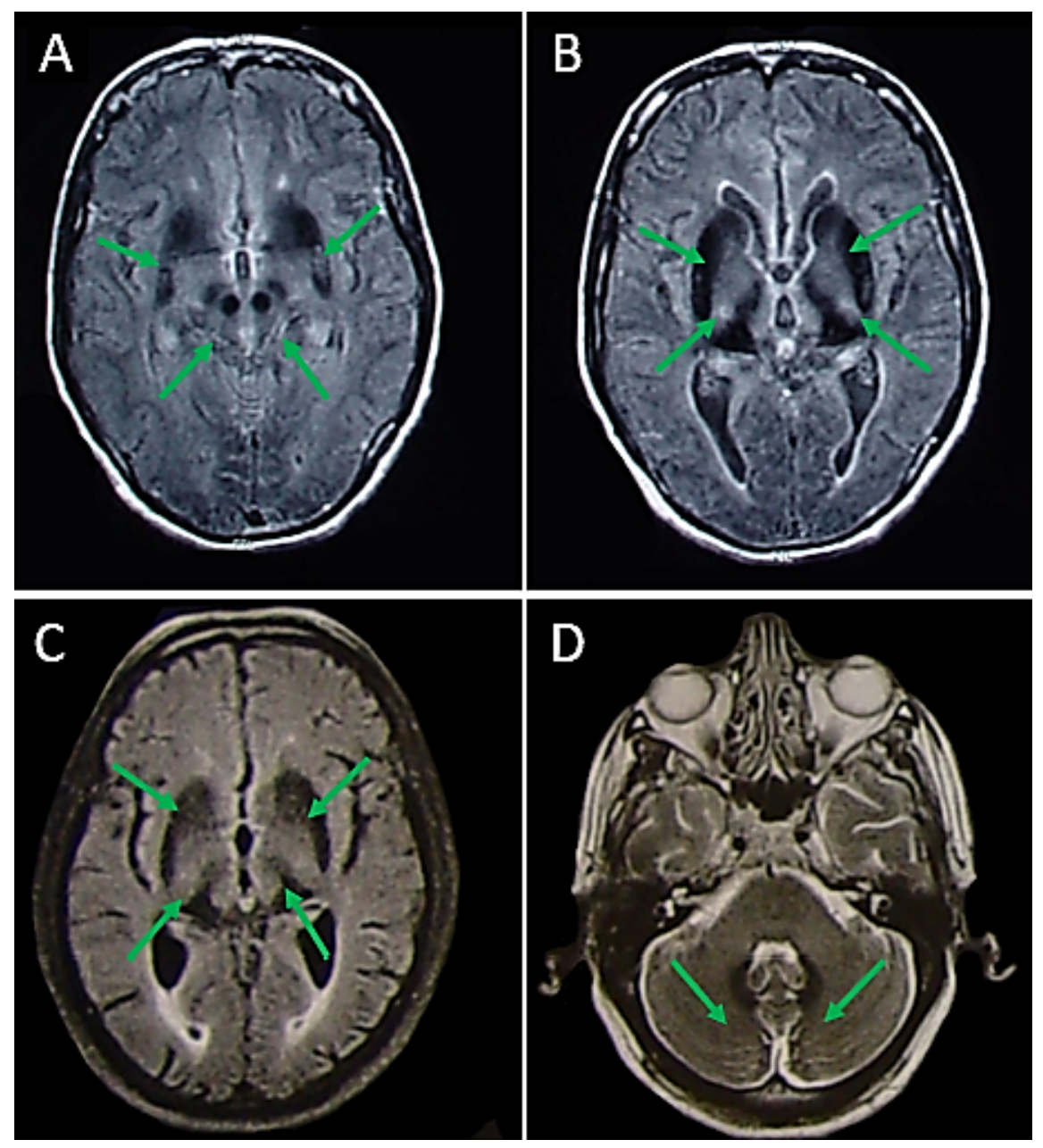

Figure legend:

Figure. A, B Magnetic resonance imaging (FLAIR) of case 1: There were low-signal intensities in the bilateral lentiform nuclei, caudate nuclei, and thalamus.

Figure. C, D Magnetic resonance imaging (T2/FLAIR) of case 2 : There were low-signal intensities in the bilateral lentiform nuclei, caudate nuclei, thalamus, and cerebellar dentate nuclei. 

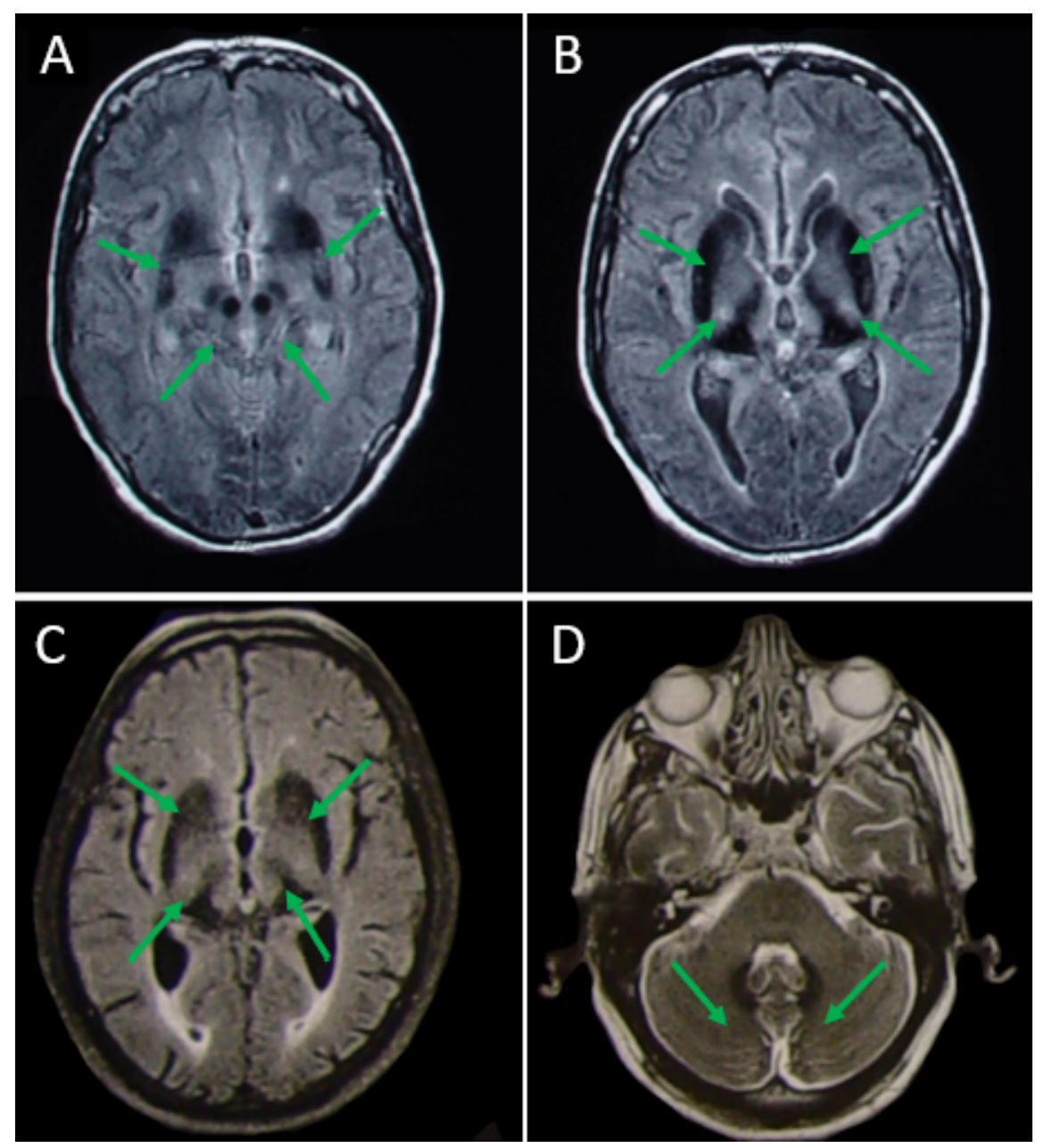